\title{
THE PHOTO-ELECTRIC THRESHOLD FOR MERCURY
}

\author{
By C. B. KAzDA
}

\section{Abstract}

In order to insure a perfectly clean surface of mercury for test, the apparatus was arranged so that carefully purified mercury flowed intermittently into a shallow iron cup and out through an overflow opening at the surface back into an electrically heated still. Direct observation showed that with such a flow the surface cleans itself of any added contamination very quickly. Also, the results for the wave-length limit were found to be independent of the rate of flow, to a rate far below that normally used, and also unaffected by the presence of gases (air, $\mathrm{H}_{2}, \mathrm{O}_{2}, \mathrm{He}$ and $\mathrm{CO}_{2}$ ) and even of traces of water vapor in the photochamber. The photo-currents to a copper oxide receiver were measured by means of a quadrant electrometer to within about 1 percent for various $\mathrm{Hg}$ lines from a Cooper-Hewitt quartz mercury arc, correction being made for stray light by means of intermediate readings. This arc operated at $92-93$ volts with $2.1 \mathrm{amp}$., was constant to 1 percent. Intensity measurements accurate to 1 percent were made by means of a vacuum thermopile connected to a d'Arsonval galvanometer (sensitivity $10^{-9} \mathrm{amp}$.) using a Hilger monochromator to disperse the light and making correction for stray light by taking readings between the lines. Plots of the ratio of corrected photo-current to corrected light intensity as a function of wave-length give the threshold at $2735 \mathrm{~A}$. The corresponding photo-electric work function is 4.52 volts. This result is free from the objections raised to those of previous observers and seems clearly to be the evaluation of an intrinsic property of mercury uncontaminated by surface impurities.

\section{INTRODLCTION}

G OLLOWING the early phase of discovery by Hertz, ${ }^{1}$ Hallwachs, ${ }^{2}$ Lenard $^{3}$ and J. J. Thomson, ${ }^{4}$ later experimentation in the field of photo-electricity has produced a good deal of conflicting evidence regarding the conditions essential to the discharge. While no attempt will be made here to summarize these investigations it may be well, however, to state that they may be divided into two groups. To the first belong those of Fredenhagen, ${ }^{5}$ Wiedemann and Hallwachs, ${ }^{6}$ Kustner, ${ }^{7}$ Hughes, ${ }^{8}$ Sende and Simon, ${ }^{9}$ and Suhrmann, ${ }^{10}$ whose experiments tend

${ }^{1}$ Hertz, Ann. der Physik, 31, 383 (1887).

${ }^{2}$ Hallwachs, Handbuch der Radiologie Vol. 3; Ann. der Physik, 33, 301 (1888).

${ }^{3}$ Lenard, Ann. der Physik 2, 359 (1900); 8, 149 (1902).

${ }^{4}$ J. J. Thomson, Phil. Mag. 48, 547 (1899).

${ }^{5}$ Friedenhagen, Phys. Zeits. 15, 65 (1914).

6 Wiedemann and Hallwachs, Ber. Deutsch. Phys. Gesell. 16, 107 (1914).

${ }^{7}$ Küstner, Ann. der Physik 46, 893 (1915).

${ }^{8}$ Hughes, Photo-electricity, Camb. Univ. Press, 1914 ed.; Nat. Res. Coun. Bull. 2, No. 10 (1921).

${ }^{9}$ Sende and Simon, Ann. der Physik 67, 697 (1921).

${ }^{10}$ Suhrmann, Ann. der Physik 67, 43 (1922). 
to throw serious doubt upon the existence of a photo-electric effect which is an intrinsic property of the material under investigation. Most of these workers conclude that the presence of impurities is an essential condition for the appearance of the photo-current. In the second group we have the investigations of Pohl and Pringsheim, ${ }^{11}$ Piersol, ${ }^{12}$ Millikan and his students, ${ }^{13}$ Welo, ${ }^{14}$ and Elster and Geitel, ${ }^{15}$ who attribute the apparent disappearance of the photo-current to a mere shift in a long wave-length limit as a result of surface impurity. Some investigators (Koppius, ${ }^{16}$ Tucker, ${ }^{17}$ and others) have shown that the threshold can thus be shifted below the limits accessible to their method of observation.

In 1905 Einstein $^{18}$ made the first direct application of the quantum theory to the photo effect and suggested the following well known equation

$$
\frac{1}{2} m v^{2}=V e=h \nu-V_{0} e
$$

where $h \nu$ is the energy absorbed by the electron from the light wave, $V_{0} e$ is the work necessary to free the electron from the metal, and $\frac{1}{2} m v^{2}$ is the energy of the emitted electron, which is measured by the product of its charge $e$ and the potential difference $V$ required to stop it. The validity of this relation between $V$ and $\nu$ has been established throughout the range of visible, ultraviolet and x-ray frequencies. The value of $h$ obtained by Millikan ${ }^{19}$ from his photo-electric experiments, is one of the most accurate yet obtained. This equation implies that there is a photoelectric threshold characteristic of the material itself; therefore an important test of the equation is to determine whether or not such an intrinsic photo-electric threshold exists, using an experimental method which is at least free from the objections made to previous experiments.

It was decided to test carefully purified mercury running in a vacuum, for by distillation the mercury can be completely freed from gases and other impurities, and a running surface remains clean. If this should give a definite threshold frequency the matter would be settled.

The method adopted to determine the long wave-length limit was that given by Millikan. ${ }^{19}$ The photo-currents per unit intensity were plotted against the wave-lengths used, and the curve extended until it inter-

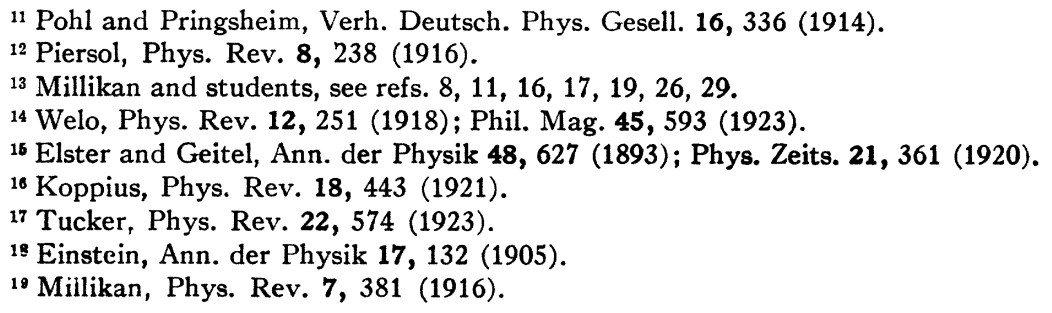


cepted the wave-length axis. This involved first the determination of the intensity distribution of the incident light and second the measurement of the photo-current as a function of wave-length.

\section{Measurement of the Energy Content of Ultraviolet Lines of the Quartz Mercury ARC}

In order to get sufficient sensitivity for such intensity measurements previous experimenters have used thermopiles in air with Paschen or Thomson Galvanometers. In this work it was decided to use the type of vacuum thermopile employed by Pettit and Nicholson ${ }^{20}$ at the Mount Wilson Observatory with a sensitivity 6 to 15 times greater, combined with a high sensitivity d'Arsonval galvanometer obtained from Leeds and Northrup. While the sensitivity $\left(10^{-9} \mathrm{amp}\right.$.) is only one tenth that of magnetically shielded galvanometers, the combination of vacuum thermopile and d'Arsonval galvanometer avoided the inevitable fluctua-

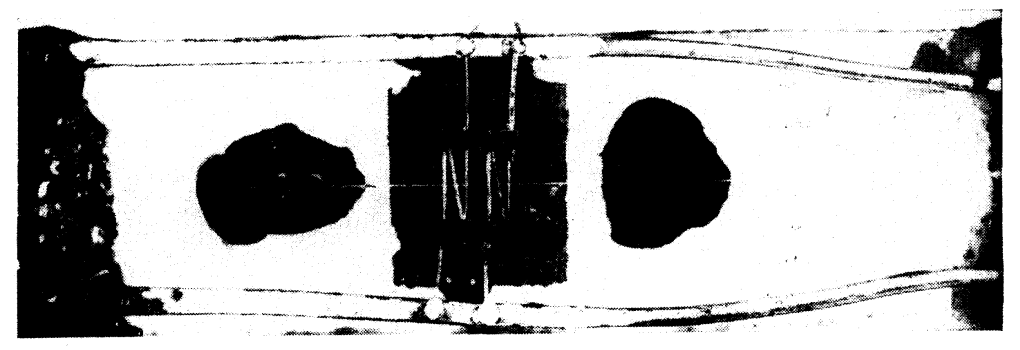

Fig. 1. Photograph of vacuum thermopile used (made by Dr. Pettit).

tions of Thomson galvanometers without loss of sensitivity. It was found necessary to suspend it by a Carman ${ }^{21}$ suspension. The telescope was about $3 \mathrm{ft}$. and the scale about $25 \mathrm{ft}$. from the galvanometer mirror. This made it unnecessary to estimate the readings to fractions of a millimeter. All of the thermopiles used were made by Dr. Pettit at the Mount Wilson Observatory. (See the article by Pettit and Nicholson for details of construction.) The photograph shown in Fig. 1 is a three fold magnification of the last thermopile used. It will be noted that the thermopile consists of four couples connected in parallel. This thermopile gave exceedingly good results and had a high sensitivity since its resistance was nearly equal to that of the galvanometer. It is mounted upon two platinum leads which are sealed into a glass plug. A glass plate is mounted below with sealing wax and a fine glass tube is fixed upon it, to support the center of the thermopile. Directly below the couples, on the

${ }^{20}$ Pettit and Nicholson, Astrophysical J. 56, 295 (1922).

${ }^{21}$ Carman, J. Opt. Soc. Amer. \& R. S. I. 6, 694 (1922). 
glass plate, is placed a piece of fluorescing paper so as to make settings possible in the extreme ultraviolet. The couples were made of bismuth and bismuth plus 5 percent tin, while the receiver was made of tin blackened on the receiving side and placed at the junction. The couples were of the compensated type, and the double deflection was used, thus eliminating the zero reading.

It should be mentioned here that the behavior of thermocouples presumably made in the same way from the same stock of materials is not uniform. Of the considerable number of couples made, some showed drift very badly and were discarded, but those used in this work showed no drift at all and remained useful for long periods of time, reproducing results which checked very well.

As shown in the article of Pettit and Nicholson, ${ }^{20}$ the sensitivity of the vacuum thermopile is practically constant for pressures below $10^{-4} \mathrm{~mm}$. The thermopile chamber in our tests was directly connected to a two stage mercury diffusion pump, backed by a Cenco Hyvac pump. There was also a liquid air trap, just beyond the thermopile cell, when the thermopile was in operation. The pressure in the cell as measured by the McLeod gauge, was always less than $10^{-5} \mathrm{~mm}$. The cell was surrounded by cotton to insure uniform temperature on all sides.

The source of light used was a Cooper-Hewitt quartz mercury arc, 100-125 volts d.c., enclosed in an asbestos lined box. The light was resolved by a Hilger monochromatic illuminator, and was then focused upon the thermopile supported inside a chamber which could be evacuated. A special carriage was made for the focusing lens which was provided with two fine screw adjustments by means of which the light could be focused on either one row of junctions or the other. By the use of both of these screw motions, a maximum reading was always obtained for each line. This accounts for the very good agreement of the individual readings obtained.

To insure constancy of operation of the mercury lamp while readings were being taken, it was necessary, in the beginning, to make a careful study of the operating conditions of the lamp. To minimize variations a resistance and reactance were connected in series with the lamp, and a 200 volt storage battery was used as source. It was found that for this lamp, when operated at 92 to 93 volts and $2.1 \mathrm{amp}$., it was possible to hold the voltage to within one volt. It might be mentioned here that it took about one hour for the lamp to reach these best conditions of operation after starting up.

The slit width used in all measurements of intensity was 20 divisions $(0.5 \mathrm{~mm})$ on the slit screw head of the Hilger Monochromator. Tucker ${ }^{17}$ 
made a measurement of the intensity for the same lines, but used a slit width of 25 divisions. The stray light which appears as a general background on photographic spectrum plates gave a measurable thermopile deflection. In general the minimum reading between two lines was taken as measuring stray light, except in the case of 2620 where weak lines were present (see Table I). The corrected value for the intensity for a given line was obtained by subtracting the nearest interpolated value for stray light from the observed intensity reading. The method is limited by the necessity for this correction which below $2300 \mathrm{~A}$ becomes very large,,$^{22}$ as well as by the fact that at $1900 \mathrm{~A}$ we begin to get strong absorption by air and quartz.

TABLE I

Typical set of readings of intensity of mercury lines from quartz arc.

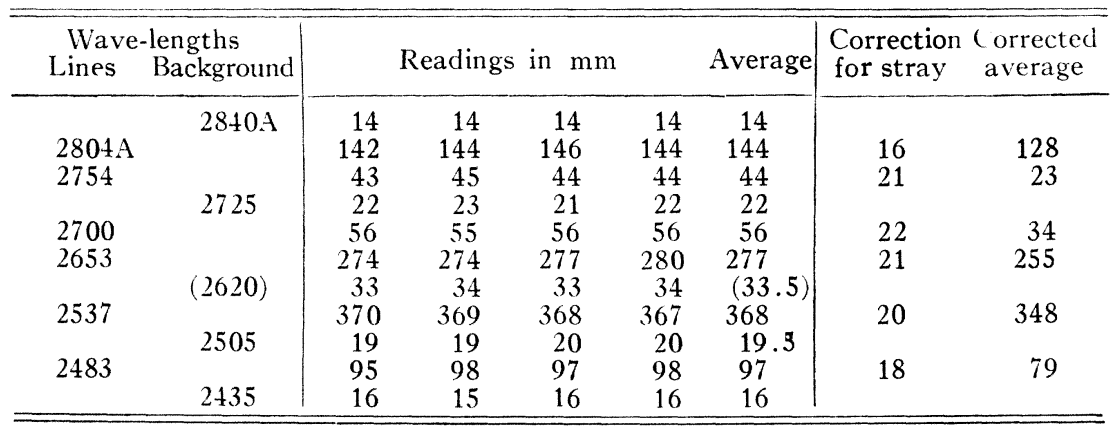

Table I gives a representative set of readings and indicates how closely the individual readings agree. This table covers only the region of wave-lengths used in the photo-electric investigation described below. It will be noticed that down to $2483 \mathrm{~A}$ the deviation from the average is less than 3 percent and in some other measurements, not here given, the agreement was even better.

Table II gives the average values of the deflections for three other sets of readings. These sets of readings were all taken under the same experimental conditions, the voltage across the lamp being 92 to 93 volts and the current $2.1 \mathrm{amp}$. The lamp could have run at higher temperatures to get greater intensity but the precautions that would have been necessary were not warranted since sufficiently large readings were obtained for this region of wave-lengths.

While the readings did change from time to time, it was found that over the intervals of time used in the latter part of the investigation, the readings remained practically constant. Readings taken one set after

${ }^{22}$ Souder, Phys. Rev. 8, 310 (1916). 
another, during the same afternoon, changed hardly at all. Over thirty sets of readings were taken.

\section{Determination of the Photo-electric Lojg Wave-Length Limit for a Clean Surface of Liquid Mercury}

Extreme care was taken to obtain pure mercury. The occluded gases were completely removed by repeated distillation in an atmosphere of mercury vapor, and the photo-current was measured as soon as the fresh surface was presented to the vacuum chamber. In this way two important objections to previous experiments are removed.

TABLE II

Three sets of average readings for intensity of mercury lines

\begin{tabular}{|c|c|c|c|c|c|c|}
\hline Wave-lengths & $9 / 8 / 23$ & $\begin{array}{l}\text { Uncorrected } \\
9 / 12 / 23\end{array}$ & $9 / 15 / 23$ & $9 / 8 / 23$ & $\begin{array}{c}\text { Corrected } \\
9 / 12 / 23\end{array}$ & $9 / 15 / 23$ \\
\hline $\begin{array}{l}2804 \mathrm{~A} \\
2754 \\
2700 \\
2653 \\
2537 \\
2483\end{array}$ & $\begin{array}{c}146 \mathrm{~mm} \\
45 \\
56 \\
280 \\
370 \\
97\end{array}$ & $\begin{array}{c}144 \mathrm{~mm} \\
44 \\
54 \\
276 \\
369 \\
97\end{array}$ & $\begin{array}{c}147 \mathrm{~mm} \\
46 \\
55 \\
283 \\
368 \\
97\end{array}$ & $\begin{array}{c}133 \mathrm{~mm} \\
23 \\
34 \\
257 \\
350 \\
82\end{array}$ & $\begin{array}{c}130 \mathrm{~mm} \\
22 \\
33 \\
255 \\
350 \\
80\end{array}$ & $\begin{array}{c}130 \mathrm{~mm} \\
23 \\
34 \\
260 \\
348 \\
81\end{array}$ \\
\hline
\end{tabular}

After being put through the wet process, the mercury was purified by the method given by Hewlett and Minchin ${ }^{23}$ which is the same as that used by Harkins ${ }^{24}$ in preparing mercury for use in isotope determinations. This method consists in evaporating mercury while bubbling air through it. The mercury was then placed in a still directly connected with the photo-electric chamber. All the apparatus had previously been carefully cleaned, washed and dried and then heated for some time while being evacuated before the mercury was finally inserted. This still was made of Pyrex glass shaped like one stage of an ordinary diffusion pump, as shown in the diagrammatic sketch (Fig. 2). The still was provided with an electric heater of which the current could be varied by external resistance to regulate the rate of evaporation. The process was carried on at low pressures since the still was directly connected to the vacuum pumps.

Continuously overflowing mercury was tried, but it was observed that the center of the surface remained stationary, while the mercury flowed out at the groove from underneath. Intermittent flow, however, was found to be effective in keeping the surface clean. Rough experiments with mercury in a glass cup with a $\mathrm{V}$ groove in it, showed that after a

${ }^{23}$ Hewlett and Minchin, Phys. Rev. 21, 388 (1905).

${ }^{24}$ Harkins and Mulliken, Jour. Amer. Chem. Soc. 44, 37 (1922). 
few overflowings, even the trace of oil which had been dropped on the surface could not be distinguished with a strong magnifying glass (and later a microscope). Fine dust could be observed to be drawn out through the groove from all parts of the surface. Thus intermittent overflow is a definite means for the removal of surface films which might possibly form when the surface is exposed to the chamber probably because the surface film is broken each time an overflow takes place. Intermittent overflow was automatically produced by the mercury dropping from the condenser. From the overflow the mercury returned to the still and was

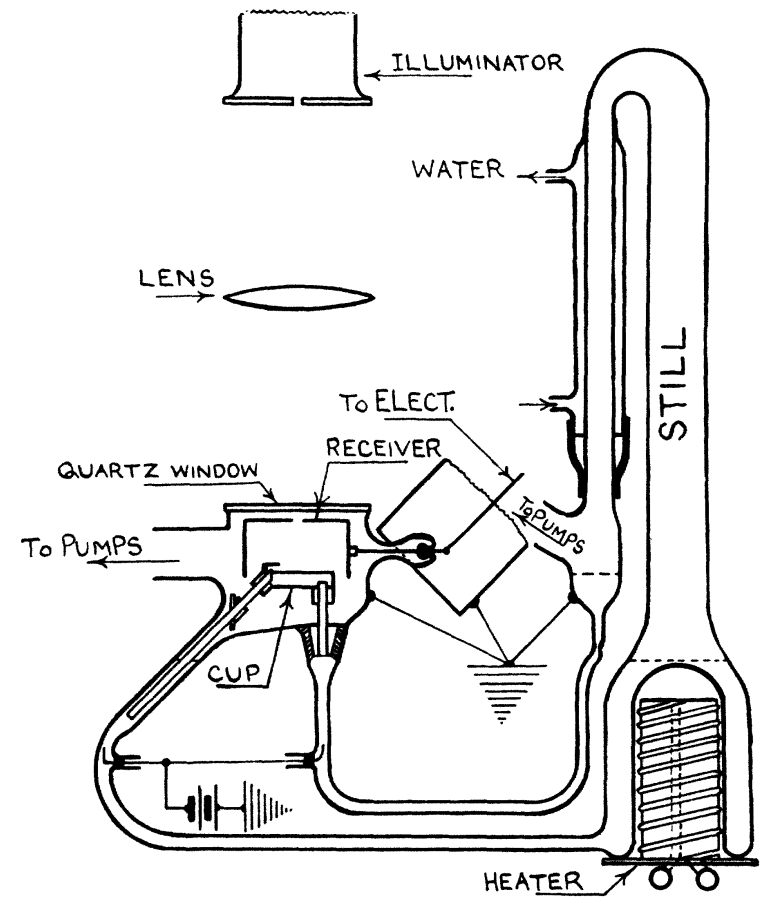

Fig. 2. Apparatus, including cup with mercury surface cleaned by intermittent overflow.

re-evaporated again. The cup itself was shallow so that a given molecule of mercury could not remain at the surface exposed to the vacuum chamber more than a second.

The attempt to take electrometer readings with overflowing mercury has been made before by $\mathrm{McGougan}^{25}$ in connection with delta rays produced by alpha rays. Others have tried it also without success. I encountered the same difficulty as they did at first, namely, an erratic behavior of the electrometer as soon as the overflow commenced. A glass cup and vertical outlet tube was being used, and the trouble seemed to be caused by friction on the glass and by separation of the mercury into

${ }^{25}$ McGougan, Phys. Rev. 12, 122 (1918). 
drops. Therefore, a change was made to the iron cup and the outlet tube of iron placed at 45 degrees. The inlet tube of iron was fitted into a ground joint in the chamber, and cemented with a special high temperature cement kindly furnished by the Mantle Lamp Company of America. (This cement was also used to attach the quartz window.) While the trouble was not eliminated, it was decreased considerably. A further search was made and at last it was found that the main source of trouble was in the mercury condensation tube since a large static charge is produced when the mercury strikes the walls of the tube. So the whole condensation tube and photo chamber were closely wrapped externally by tin foil which was then grounded. The erratic behavior of the electrometer ceased entirely and results followed quickly.

The vacuum pumps were directly connected to the photo chamber and run continuously during the experiment. The pressure was read by a McLeod gauge and was never more than $10^{-5} \mathrm{~mm}$. This does not include the pressure of the mercury vapor which was of the order of $10^{-3} \mathrm{~mm}$ at room temperature. A liquid air trap was situated near the photo chamber. Air was never introduced except through a number of drying tubes of calcium chloride and phosphorous pentoxide.

As shown in the diagram, the cup is surrounded by a copper oxide receiver. A slit in the top permits the illumination to reach the mercury in the cup. The slit is of such dimensions as not to be touched by the concentrated light from the illuminator. The same condensing lens was used as in the intensity measurements. The thermopile chamber and the photo chamber were side by side and the lateral screw motion on the holder of the condensing lens was large enough to enable the light to be focused on either chamber.

The monochromatic illuminator was mounted so that both collimator and telescope were in the same vertical plane. The lamp was mounted on a special carriage and rigidly connected to the illuminator, so that the light could be shifted from one chamber to the other by motion along a guide. This moving element being heavy was counterweighted from the ceiling so that the shift could be made easily and quickly.

The photo-current readings were made with a Cambridge and Paul Dolezalek quadrant electrometer. One pair of quadrants was grounded while the other pair was connected to the receiver. The sensitivity was $1250 \mathrm{~mm}$ per volt with the scale at $150 \mathrm{~cm}$ distance for a needle voltage of 92 . The photo surface was kept sufficiently negative to ground to insure maximum readings.

The electrometer readings were taken for periods of 40 seconds or more for small currents and 8 seconds for large currents, but were all 
reduced to an 8 second basis. The time interval was measured by means of a metronome which was frequently checked against a stop watch and was controlled by means of a camera shutter fitted to the outlet hole in the box containing the mercury lamp, between it and the illuminator. Correction for the electrometer leak, which was always very small and taken with each observation, was always applied to the reading. The minimum readings between lines were taken as in the intensity measurements and the same procedure was followed in correcting for stray light. This is very important here for the stray light may be composed of wave-lengths above and below that of the line itself.

As the heater on the mercury still was started at the same time as the lamp, the mercury was evaporating for some time before readings were begun. A set of intensity readings for the various wave-lengths was taken first, followed by a set of photo-current readings. Care was taken to get a maximum for each reading and three or four readings were taken for each line.

\section{Experimental Results}

Table III gives a representative set of detailed photo-current readings. The maximum deviation from average in Table III is less than 3 percent. Thus the accuracy of measurement of the photo-current is about the same as that of the light intensity.

TABLE III

\begin{tabular}{ccc|rrrr|rc}
\hline \hline \multicolumn{2}{c}{$\begin{array}{c}\text { Wave-lengths } \\
\text { Lines }\end{array}$} & Bet. lines & $\begin{array}{c}\text { Time } \\
\text { (sec.) }\end{array}$ & \multicolumn{7}{c|}{$\begin{array}{c}\text { Reading in mm } \\
2\end{array}$} & 3 & Aver. & Stray* $^{*} \begin{array}{c}\text { Correct.* } \\
\text { av. }\end{array}$ \\
\hline $2804 \mathrm{~A}$ & & 240 & 0 & 0 & 0 & 0 & 0 & 0 \\
& $2770 \mathrm{~A}$ & 80 & 0 & 0 & 0 & 0 & 0 & $\ldots .$. \\
2754 & & 80 & 10 & 10 & 10 & $1.0^{*}$ & 1.0 & 0 \\
& 2725 & 40 & 17 & 17 & 16 & $3.6^{*}$ & 3.6 & $\ldots .6$ \\
2700 & & 8 & 12 & 12 & 12 & 12.0 & 6.2 & 5.8 \\
2653 & & 8 & 129 & 125 & 128 & 127.0 & 18.0 & 109.0 \\
2537 & $(2620)$ & 8 & 38 & 38 & 38 & $(38.0)$ & & \\
& & 8 & 444 & 465 & 461 & 456.7 & 32.0 & 424.7 \\
2483 & 2505 & 8 & 32 & 33 & 32 & 32.3 & 32.3 & \\
& 2435 & 8 & 166 & 167 & 164 & 165.7 & 32.0 & 133.7 \\
\hline
\end{tabular}

* Reduced to a time interval of $8 \mathrm{sec}$.

No mention is made by most investigators of stray light corrections to their readings but to show the magnitude of this correction, the values are here given for both the corrected and uncorrected readings. Table IV gives the photo-current readings corresponding to the intensity measurements in Table II. The thirty sets of readings which were taken all closely check with these three sets. 
In every case, a deflection was obtained for $2700 \mathrm{~A}$ but none whatever for the much more intense line $2804 \mathrm{~A}$, even when exposed for several minutes. In the case of $2754 \mathrm{~A}$, the very small deflection obtained over a period of $40 \mathrm{sec}$. was just equal to the deflection given by the stray light for that line, so its net reading was zero. These readings were the same even when the rate of overflow was decreased somewhat. It seems certain, therefore, that the surface could not be contaminated in any way by this extremely short contact with the vacuum chamber.

TABLE IV

\begin{tabular}{c|ccc|ccc}
\hline \hline Wave-lengths & \multicolumn{3}{|c|}{ Uncorrected } \\
& $9 / 8 / 23$ & $9 / 12 / 23$ & $9 / 15 / 23$ & & \multicolumn{3}{c}{$\begin{array}{c}\text { Corrected } \\
9 / 8 / 23\end{array}$} \\
\hline 28/12/23 & $9 / 15 / 23$ \\
\hline 2754 & $0 \mathrm{Am}$ & $0 \mathrm{~mm}$ & $0 \mathrm{~mm}$ & $0 \mathrm{~mm}$ & $0 \mathrm{~mm}$ & $0 \mathrm{~mm}$ \\
2700 & 0.8 & 0.7 & 1.0 & 0 & 0 & 0 \\
2652 & 12.5 & 12.0 & 12.0 & 5.2 & 5.5 & 5.8 \\
2537 & 128.0 & 126.0 & 127.0 & 109.0 & 108.0 & 109.0 \\
2483 & 451.0 & 450.5 & 456.7 & 420.0 & 425.0 & 424.7 \\
\hline
\end{tabular}

As a further test, air, hydrogen, oxygen, helium and carbon dioxide were introduced into the chamber. No extended effort was made to purify them but they were very carefully dried beforehand. Under the conditions of this method with a rapidly changing surface they were without effect upon the limit. Even a trace of water vapor at rapid intermittent flow was without effect. These results could be reproduced at will.

As mentioned before, the long wave-length limit or threshold is obtained by plotting the photo-current per unit intensity against the wa ve-length or frequency, and extrapolating the curve to the intersection with the latter axis. Since the strong line $2804 \mathrm{~A}$ gives no deflection and the much weaker line $2700 \mathrm{~A}$ (see Table I) gives a good deflection, it is certain that the limit must fall between them.

It must be borne in mind that this intersection is governed by the points near the axis and not at all by points farther away from the axis, and therefore, it is useless to make measurements far from the axis. The curves were drawn on a large sheet of cross-section paper 20 inches wide and 40 inches long and use was made of regulation draftsmen's long flexible guides and weights and the natural position of the guides in every case governed the direction. This insures a uniform method of drawing the curves because the guides are in contact with the paper at only a few places so that the friction of the guides with the paper is small.

Since the individual curves all fall very close together, in Fig. 3 are given only the curves obtained by plotting the ratio of the final average 
values of the photo-current readings and of the corresponding intensity readings, both uncorrected and corrected, against the wave-length. The individual curves, on a large scale, show a variation of plus or minus $10 \mathrm{~A}$ for the threshold value, for both corrected and uncorrected. It will be observed that the photo-current, due to stray light, decreases rapidly as the wave-length increases beyond $2750 \mathrm{~A}$ dropping to zero at $2770 \mathrm{~A}$. while the intensity reading (Table I) is still $144 \mathrm{~mm}$ at $2804 \mathrm{~A}$. This indicates that, fortunately, the stray light here is largely of longer wavelength and hence does not excite photo-emission. As a result the average curve for the uncorrected effect gives practically the same threshold value

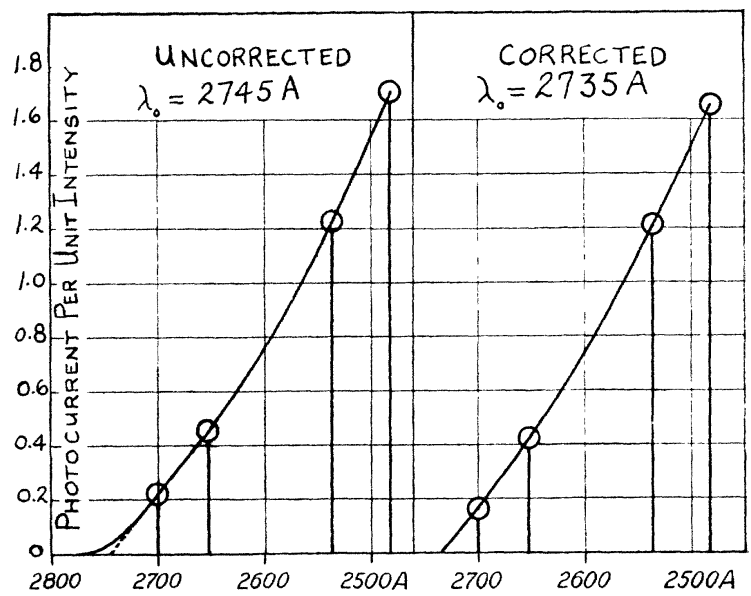

Fig. 3. Photo-current from clean mercury surface as a function of wave-length, corrected for stray light and uncorrected. Value for 2754, uncorrected, is .019.

$(2745 \mathrm{~A})$ as the average curve for the corrected effect. Nevertheless, as emphasized by Professor Millikan, ${ }^{19}$ to determine the threshold accurately it is important to make the correction for stray light.

\section{Discession}

The only other data that could be found for mercury were some given by Derieux ${ }^{26}$ and a curve by Pringsheim. Derieux states that "the drops of mercury were sensitive to line $2536 \mathrm{~A}$ but not to line $3126 \mathrm{~A}$ and the lines between were too weak to give any readings." The curve by Pohl and Pringsheim is given on page 76 of Hughes' book on photo-electricity . The last reading is for line 2536A. Klages ${ }^{27}$ made an attempt to work on mercury in 1909 but does not.mention the limit.

\footnotetext{
${ }^{26}$ Derieux, Phys. Rev. 11, 276 (1918).

:7 Klages, Ann. der Physik 31, 343 (1910).
} 
Tests with water vapor showed that it has a marked effect upon stationary mercury even though present in extremely small amounts. The effect of a trace of water vapor at $10^{-5} \mathrm{~mm}$ pressure was very noticeable. It raised the long wave-length limit. When a greater amount of water vapor was admitted the limit was pushed below the lowest value the apparatus was capable of measuring. That a film of water vapor can exist on mercury and have a noticeable effect has also been found by Iredale ${ }^{28}$ in connection with surface tension measurements at pressures of the order of $10^{-5} \mathrm{~mm}$.

It is entirely possible that water vapor is responsible for much of the non-agreement in previous work in this field. It was also found that when once admitted water vapor is extremely hard to get rid of. However, in this experiment, as stated above, the presence of a trace of water vapor did not affect the readings when the surface was changing rapidly.

It seems clear that the present method for determining the threshold value for mercury is free from the chances of contamination present in previous experiments. The measurement of photocurrent at the instant a clean photosurface is presented, has not been tried before.

Millikan ${ }^{29}$ has pointed out that the necessary conclusion from the constancy of stopping potentials is that each metal has a definite long wave-length limit and that furthermore, the photo-electric and thermionic work required to remove an electron from the surface should agree. The value of the photo-electric work function computed from the measured long wave-length limit is

$$
h \nu_{0}=300 h c / e \lambda_{0} \times 10^{-8}=12345 / \lambda_{0}=4.52 \text { volts }
$$

assuming $e=4.77 \times 10^{-10} ; h=6.55 \times 10^{-27} ; c=3 \times 10^{10} ; \lambda_{0}=2735 \mathrm{~A}$.

In conclusion, I wish to thank Professor Millikan for suggesting this work with flowing mercury and for putting the facilities of the laboratory at my disposal.

Norman Bridge Laboratory of Physics,

California Institute of Technology,

Pasadena, California,

November 20,1923*

${ }^{28}$ Iredale, Phil. Mag. 45, 1088 (1923); 48, 177 (1924).

${ }^{29}$ Millikan, Phys. Rev. 18, 236 (1921).

* Received Jan. 20, 1925; revised Aug. 20, 1925.-Ed. 


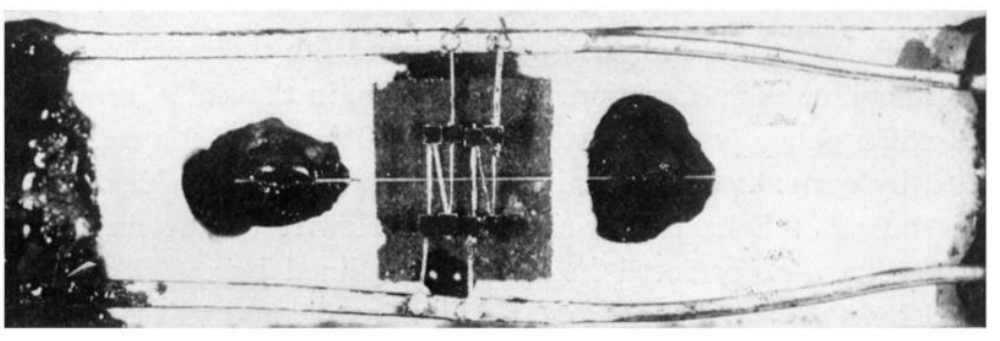

Fig. 1. Photograph of vacuum thermopile used (made by Dr. Pettit). 